un peu en aval du premier ouvrage, et on essaya vainement de constituer un nouveau barrage en garnissant l'intervalle avec des fascinages et des sacs de sable. Mais on ne tarda pas à reconnaître l'inutilité de ce travail, et on l'abandonna. En juillet 1905 , on battit à nouveau des pieux en $\mathrm{C}$, et on los entrelaca avec des fascines de manière à constituer un harrage oblique destiné à rejeter les eaux du Colorado dans une branche du fleuve située à la gauche de l'ile, mais des affouillements ne tardèrent pas à se produire et fon dut abandonner à nouveau les travaux.

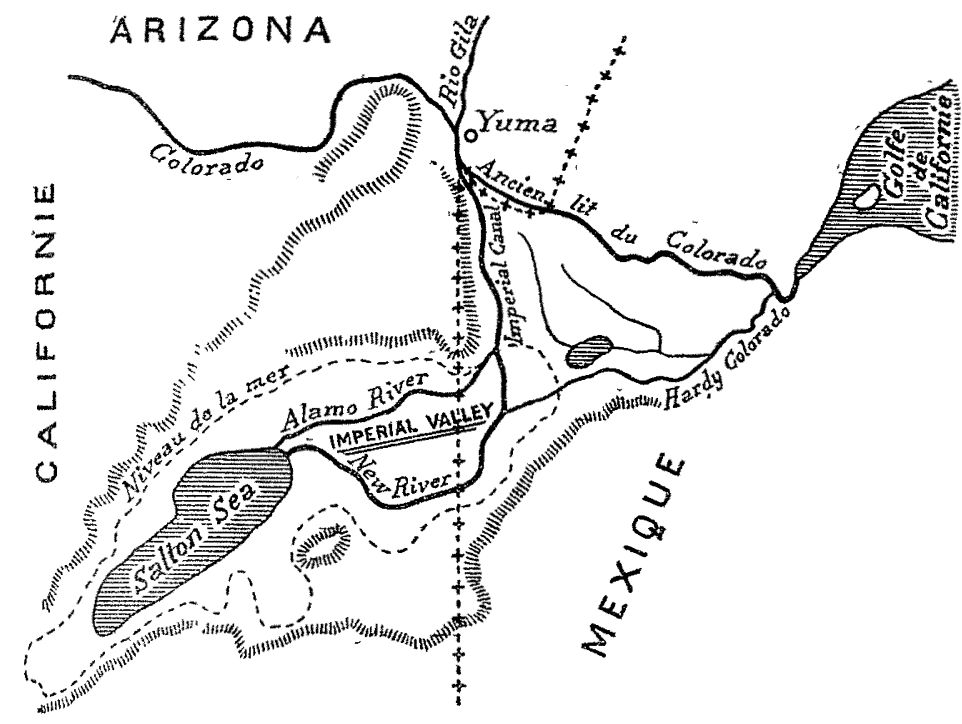

Pri 1 - Carle tu salton Sea, de I'Impérial. Vitley et de I embonchure du Coloradó

Un quatric̀me essai fut tenté en novembre 1905. On procéda à la création d'un nouveau barrigre en D, dont la fondation étalt constituée par des fascmages chargés de pierres et traversés par des pieux. Il $y$ avait à peine un mois que ce barrage otait établi qu'il fut emporté par une crue du Rio Sila. Tout le Colorado se déversa alors dans l'imperial Canal, se procipitant vers le Salton Sca, pendant que l'ancion lit devenait complètement ì sec.

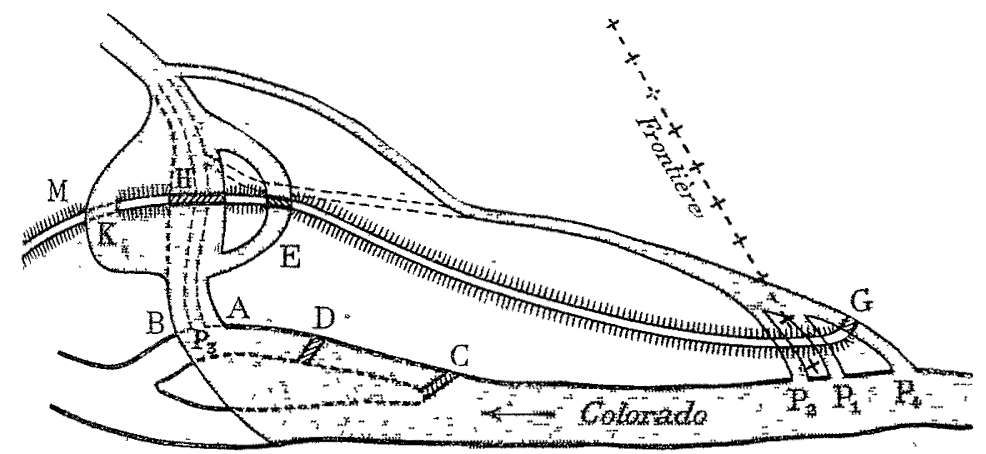

Fig 2. - Emplacements des prises d'eau et des travaux exćcutés pour ramener le Colorado lans son ancien ht.

On imagina alors de créer en E une dérivation et d'y élablir un barrage en charpente, large de $61 \mathrm{~m}$., et muni de vannes. On pensait ainsi faire passer, pendant les basses eaux, le débit du Colorado dans cette dérivation, de manièrè átablir à son aise un barrage solide en travers de la brèche. On créa aussi, toujours dans le même but, une quatrième prise d'eau $P_{4}$, en amont des deux premières, et on construisit en $\mathrm{G}$ un barrage en béton, muni de 11 vannes métalliques Taintor, de $3^{\mathrm{m}} 66$ de large et de $3^{\mathrm{m}} 05$ de haut. La prise d'eau no 3 avait alors 549 mètres de largeur.

La Compagnie du Southern Pacific, qui était obligée de déplacer ses voies le long du Salton Sea, et dans l'Imperial était , devant l'envahissement des eaux, et dont le trafic était sérieusement menacé dans cette région, prit alors la direction des travaux. En août 1906 , pendant une période de basses eạx, elle fit procéder, avec grande activité, à l'établissement d'un barrage en remblais rocheux, établi en $H$ au travers de lá bréché. Ce barrage se continuait en $M$ par une digue de $600 \mathrm{~m}$. de longueur. On réussit alors à faire passer l'eau par la dérivation E. Le succès paraissait assuré, mais, sous l'influence d'une nouvelle crue, 36 mètres du barrage $\mathrm{E}$ furent emportés, et le libre passage était à nouveau ouvert au Colorado vers l'Imperial Valley.

La digue récemment construite ayant tenu bon, on sóngea à clore la dérivation $\mathrm{E}$, et, pour cela, on y construisi conséculivement trois barrages parallèles, "chacun d'eux supportant une partie de la charge d'eau. Ge sixième essai de clôture réussit provisoirement, et, le 4 novembre 1906, le Colorado retournait vers le golfe de Californie. Mais, un. mois après, une brèche se déclarait en $\mathrm{K}$ dans la digue $\mathrm{M}$, èt le Colorado retournait à nouveau vers le Salton Sea.

Deux millions de dollars avaient déjà été dépensés vainement, et des pertes incalculables causées aux colons de l'Imperial Valley. La siluation paraissant désespérée, M. Hariman, président de la southern Pacific $\mathrm{C}^{\circ}$, télégrapha au Président des litats-Unis pour solliciter le concours du Gouvernement fédéral. Celui ci prit alors des mesures en conséquence.

Entre temps, la Southern Pacific Co,profitant d'une courte période de hasses eaux, reprit activement les travaux. Deux paires de deux rangées de pieux furent battues et garnies d'enrochements et de remblais. Enfin, après diverses péripéties, le Colorado reprenart à nouveau són ancien lit, le 11 lévlır 1907.

Le 2 avril 1907, a eu lieu une forte crue, mais la digue et les barrages ont résisté. Comme les ingénıurs ont désormals toute latitude pour consolider les travaux exécutés, ll y a tout lieu de crorre que le Colorado est condamné à retourner d'une manière définitive vers le golfe de Californie, à li) condition toutefois quon le surveille avec soin

M. P.

\section{THÉORIE DU DÉVERSOIR}

Récemment, M. Boussinesq a présenté a l'Académie des Sciences deux notes sur la théorie de l'écoulement de l'eau par déversoir. Ces deux notes sont d'ailleurs la suite d'ure sérıe de notes que ce savant a donné antérieurement sur cette question.

Le déversorr etant l'un des modes de jaugeages les plus èmployés, et des plus commodes, pour la mesure du rendement des moteurs hydrauliques, 11 nous a paru intéressant de reprodure ici les notes de M. Boussinesq, d'après les Comptes Rendus de l'Academie, et nous publions aujourd'hui les deux premières de ces notes.

Sur la théorie de l'écoulement par un déversoir en mince paroi, saus contraction latérale, et avec la nappe déversante libre en dessous ( ${ }^{*}$.

De tous les problemes usuels de l'hydraulique, le plus rebelle à la théoric, jusqu'à présent, a été celui de l'écoulement par les déversoirs, surtout par les déversoirs en mince paroi, malgré la possibilité d'y supposer établie la permanence du régime, d'y négliger les frottements comme dans les autres phénomènes de contraction rapide de veines et, par suite, d'y appliquer le théorôme de Bernouilli, qui y donne:

$$
\frac{V^{2}}{2 g}+\frac{p}{\rho^{2}}+z=h
$$

(*) Note du 4 juillet 1887 .

A titre documentaire, nous signalerons à nos lecteurs qu'ils pourront trouver dans les comptes rendus de l'Académie des Sciences de $186 \mathrm{r}$, page 12 , une note de M. BraschanN établissant sa formule, "qui est tres employée en. Allemagne: 
₹ désignant la densité, ou $\rho g$ le poids spécifique du liquide, z l'altitude de chaque point au-dessus du niveau du seuil, $V$ la vitesse qu'on y observe, $p$ l'excédent de la pression qui s'y exerce sur celle de l'atmosphère, enfin $h$ l'altitude, constante et donnée, de la surface libre à quelque distance en amont du déversoir, dans les régions d'où partent avec une vitesse supposée négligeable tous les filets fluides.

Cependant Bélanger, en considérant le cas particulièrement simple d'un seuil épais et évasé à son entrée, audessous duquel les filets fluides, tous rectilignes et horizontaux sur une petite longueur, donneraient lieu à une distribution hydrostatique des pressions, a reconnu, par comparaison avec l'expérience, que la hauteur $\eta$ de la lame d'eau paraissait s'y régler de manière à rendre maximum le débit $q$ par unıté de longueur du déversoir, tel qu'il se déduit alors de la formule (1); car $p$ s'y réduisant à $p g\left(r_{1}-z\right)$, la formule (I) y donne d'abord:

puis :

$$
V=\sqrt{2 g(h-\eta)}
$$

$$
q=\int_{0}^{n} V \mathrm{~d}_{i}=r_{i} V \overline{2 g\left(h-\eta_{i}\right)}
$$

expression nulle aux deux limites $\eta=o, \eta=h$ et maxima pour $\eta=\frac{2}{3} h$, cas où elle atteint la valeur :

$$
\frac{2}{3 \sqrt{3}} h \sqrt{2 g h}=0,385 h \sqrt{2 g h}
$$

à peu près conforme aux résultats de l'observation.

Et l'on sent, en effet, que le régime d'un cours d'eau, dans un lit déterminé, ne doit devenir stable que lorsque la masse fluide y coule partout le plus bas possible, c'est-à-dire, en particulier, quand, à l'amont d'un déversoir, le niveau garde la hauteur strictement suffisante pour que les vitesses $V$ régies par la formule (I) fournissent le débit obligé du cours d'eau. L'altitude d'amont $h$ est donc minima pour une valeur donnée de $q$, ce qui revient évidemment à prendre $q$ maximum, si ce n'est au contraire la hauteur $h$ que l'on donne.

Le principe de débit maximum dont Bélanger a eu l'idée étant ainsi regardé comme acquis, je me propose, dans cette note, d'en faire l'application aux déversoirs en mince paroi, et à nappe libre, c'est-à-dire en contact avec l'air atmosphérique, intérieurement comme supérieurement, pour le cas très usuel oú il n'y a pas de contraction latérale, le déversoir s'étendant par exemple à toute la largeur du réservoir du lit d'amont, et les molécules fluides se mouvant de la même manière dans les plans verticaux perpendiculaires à l'arête du seuil. Alors, il y a, un peu en aval de de celui-ci, une section contractée, sensiblement verticale, où tous les filets fluides peuvent être supposés parallèles, mais non plus rectilignes, comme au-dessous d'un seuil horizontal épais; car la pression $p$, nulle sur la face inférieure de la nappe de déversement, ne varie plus d'après la loi hydrostatique entre cette face et la face supérieure.

L'hypothèse la plus simple qu'on puisse faire sur les formes respectives des filets, près de la section contractée est, vu la courbe plus forte des filets inférieurs, qu'ils y admettent tous un centre commun de courbure, et, par suite, un rayon $R$ exprimé par $R_{0}+z-\varepsilon$.
Si $R_{\boldsymbol{\theta}}$ désigne le rayon de courbure le plus bas, et $\varepsilon$ l'altitude de ce filet au-dessous du seuil, c'est-à-dire le relèvement maximum total éprouvé à partir du seuil par la face inférieure du jet liquide formée de particules qui ont glissé contre la surface d'amont du déversoir et qui, par conséquent, à l'instant où elles quittent le seuil, possèdent une vitesse ascendante, verticale même dans le cas d'un déver. soir vertical. Ce relèvement $\varepsilon$ est évidemment fonction de l'inclinaison du déversoir, et je supposerai son rapport à la hauteur $h$ de charge fourni directement, comme donnée pouvant suppléer à la connaissance de l'inclinaison dont il s'agit.

Cela admis, le long d'un chemin $\int \mathrm{d} z$, normal aux filets fluides dans la section [contractée, l'accélération des molécules aura, pour projection $w^{\prime}$ sur ce chemin, sa composante centrifuge $-\frac{V^{2}}{R}$, ou $-\frac{V^{2}}{R+z-\varepsilon}$ et l'équation connue $\frac{x}{p} \frac{\mathrm{d} p}{\mathrm{~d} z}=-g-w$ ' y sera :

$$
\frac{1}{\rho} \frac{\mathrm{d} p}{\mathrm{~d} z}=-g+\frac{V^{2}}{R_{0}+z-\varepsilon}
$$

Substituons y la valeur de $\frac{1}{\rho} \frac{\mathrm{d} p}{\mathrm{~d} p}$ que donne la différentiation de ( 1 ), et qui est :

$$
-g-V \frac{\mathrm{d} V}{\mathrm{~d} z}
$$

11 viendra, en multipliant par $\left(R_{0}+z-\varepsilon\right)$, divisant par $V$ et transposant :

$$
\frac{\mathrm{d}}{\mathrm{d} z}\left[V\left(R_{0}+z-\varepsilon\right)\right]=o
$$

ou, par une intégration immédiate dans le plan de la section contractée :

$$
V\left(R_{0}+z-\varepsilon\right)=\text { Constante }
$$

relation qui, jointe à (I), tiendra évidemment lieu de la précédente (2). Donc, aux points où ils traversent la section contractée, les filets fluides possèdent (comme dans les tourbillons à axe vertical) des vitesses $V$ inverses de la distance $\left(R_{0}+z-\varepsilon\right)$ à leur centre de courbure. Or, pour $z=\varepsilon, c^{\prime}$ est-à-dire à la surface libre inférieure, la pression $p$ s'annule, et l'équation (1) donne $V=\sqrt{2 g(h-\varepsilon)}$, valeur qui, portée avec celle $\varepsilon$ de $₹$ dans la formule (3), y détermine la constante. Il vient, au lieu de (3), en divisant par $R_{\mathrm{o}}+z-\varepsilon$ :

$$
V=V \overline{2 g(h-\varepsilon)} \frac{R_{0}}{R_{0}+z-\varepsilon} .
$$

Et comme d'ailleurs cette valeur de $V$ doit, à la surface supérieure, où $p=o$, se réduire d'après (I) à $\sqrt{2 g(h-i)}$, il en résulte une relation entre l'épaisseur $\eta=z-\varepsilon$ de la nappe déversante et le rayon $R_{\mathbf{0}}$ de courbure du filet inférieur. Si l'on prend, en effet, $z=\varepsilon+\eta$ dans (4) et par suite :

$$
V=\sqrt{2 g\left(h-\varepsilon-r_{i}\right)}
$$

on obtient la proportion :

$$
\frac{V \overline{h-\varepsilon-r_{1}}}{V \overline{h-\varepsilon}}=\frac{R_{0}}{R_{0}+\eta}
$$


d'où :

$$
R_{0}=\eta_{1} \frac{V \overline{h-\varepsilon-\eta}}{\sqrt{h-\varepsilon-\sqrt{h-\varepsilon-\eta}}}
$$

Appelons, pour abréger, $\mathbf{I}-k^{2}$ le rapport de la hauteur $\eta$ de la section contractée à la hauteur $h-\varepsilon$, du niveau d'amont au-dessus du bord inférieur de cette section, ou posons :

$$
k=\sqrt{\mathrm{I}-\frac{\gamma}{h-\varepsilon}} \quad r_{i}=\left(\mathrm{r}-k^{2}\right)(h-\varepsilon)
$$

et par conséquent, vui (5):

$$
R_{0}=\frac{k\left(\mathrm{I}-k^{2}\right)}{\mathrm{I}-k}(h-\varepsilon)=\left(k+k^{2}\right)(h-\varepsilon)
$$

Le relèvement $\varepsilon$ du bas de la nappe étant sensé donner (avec la hauteur $l i$ de charge), l'expression (7) de $R_{0}$, celle (4) de. $V$ et enfin l'expression de $p$, rćsultant de l'équation ( $\mathrm{I}$ ), ne dépendront plus que de l'épaisseur encore inconnue $\eta_{\text {q }}$ de 1a nappe sur la section contractée, c'est-à-dire, en définitive, du nombre $k$; et cette valeur de $p$. s'annulera bien aux deux limttes $i=\varepsilon$ et $z=\varepsilon+\eta$ d'après la manière dont on én a déduit de (I), à ces limites, les valeurs simples $\sqrt{2 g(h-\varepsilon)}$ de $V$ indiquées par les formules (4), (5) ou (7)

Il est bon d'observer que ta relation (2), où $V$ décroit lorsque zgrandit, fournit une dérivée $\frac{d p}{d z}$ elle-même décroissante quand $z$ croît et que, $p$ s'annulant aux deux limites $i=z, z=\varepsilon+\eta$, sa dérivée s'annule dans l'intervalle, de manière à être d'abord positive, puis négațire, ou à rendre la pression $p$ positive comme dans tout l'intérieur de la section contraçtée.

La valeur maxima de $p$ sè produit de la sorte d'après (2) pơulr :

$$
V^{2}=g\left(R_{0}+i-\varepsilon\right)
$$

c'est-à-dire; vư (4), pour :

$$
z-\varepsilon=-R_{0}+\left[2 R_{0} \cdot(h-\varepsilon)\right]^{\frac{1}{3}}
$$

et il faut, en vertu de (I) :

$$
p g\left(h-\varepsilon+R_{0}\right)=\frac{\mathfrak{3}}{2} p g\left[2 R_{0}^{2}(h-\varepsilon)\right]^{\frac{2}{3}}
$$

Si nous introduisons, dans ces résultats, l'expression (7) de $R$, nous aurons, pour calculer cette valeur de $z-\varepsilon$, ẹt la pression maxima $p$ correspondante, les deux formules :

$$
\begin{gathered}
\left.z-\varepsilon=2\left[\left(\frac{k+k^{2}}{2}\right)^{\frac{2}{3}}-\frac{\left(k+k^{2}\right)}{2}\right)\right](h-\varepsilon) \\
p=\left[1-3\left(\frac{k+k^{2}}{2}\right)^{\frac{2}{3}}+2\left(\frac{k+k^{2}}{2}\right)\right] p g(h-\varepsilon)
\end{gathered}
$$

Il ne reste plus qu'à déterminer l'inconnu $p$ de manière à rendre maximum le débit :

$$
q=\int_{\varepsilon}^{n+s} V \mathrm{~d} z
$$

A cet effet, la valeur (4) de $V$ multipliẻe par dz, et intégrée de $z=\varepsilon \grave{a} z=\varepsilon+\eta$ donne, en substituant à $\eta$ et à $R_{0}$ leurs valeurs (6) et $(7)$ :

$$
q=\left\{\begin{array}{l}
\sqrt{2 g(h-\varepsilon)} R_{\theta} \log \frac{R_{0}+\eta}{R_{0}} \\
\sqrt{2 g}(h-\varepsilon)^{\frac{3}{2}}\left(k+k^{2}\right) \log \frac{1}{h}
\end{array}\right.
$$

Le débit $q$ par unité de longueur du dẻversoir se trouve donc, quand on fait varier $k$ proportionnel à la fonction $\left(k+k^{2}\right) \log \frac{t}{k}$ essentiellement positive entre les deux limites $k=0$ et $k=\mathrm{I}$; maìs nulle à ces deux limites, et par conséquent maxima pour une certaine valeur intermédiaire de $k$. La dérivée de cette fonction est, après suppression du facteur positif $(\mathrm{I}+2 k)$ :

$$
\log \frac{1}{k}-\frac{1+k}{1+2 k}
$$

quantité ayant, pour $k$ positif, sa propre dérivée $\frac{1+3 k+4 k^{2}}{k(1+2 k)^{2}}$ essentiellement négative; en sorte qu'elle ne s'annule ou donne bien à la fonction un maximum unique parfaitement déterminé. Or, la valeur de $k$ pour laquelle on a ainsi $\log \frac{1}{r}=\frac{1+k}{1+2 k}$ est de 0,46854 ; et il en résulte pour $\eta, R_{0}, q$, d'après $(6),(\zeta)$ et (q) les váleurs :

$$
\left.\begin{array}{rl}
r_{i} & =0,7805(h-\varepsilon), \\
R_{0} & =0,688 \mathrm{I}(h-\varepsilon) \\
q & =0,5 \mathrm{2} \cdot 6 \sqrt{2 \dot{g}}(h-\varepsilon)^{\frac{x}{2}}
\end{array}\right\}
$$

Quant à la section maxima dans la section contractée, et à la hauteur $\mathfrak{i}-\varepsilon$ où cette pression se produit, elles sont d'après les formules $(8)$ :

$$
\begin{aligned}
& p=0,215 \mathrm{r} g(h-\varepsilon) \\
& i-\varepsilon=0,2939(h-\varepsilon) !
\end{aligned}
$$

Celle des formules précédentes qui importe le plus dans la pratique est l'expression ( 1 1 ) du débit qà laquelle on peut donner la forme:

$$
q=m h \sqrt{2 g h}
$$

employẻe depuis Du Buat par les hydrauliciens, en attribuant aux coefficient de dépense $m$ la valeur :

$$
m=0,5216 \cdot\left(\mathrm{I}-\frac{\varepsilon}{h}\right)^{\frac{3}{2}}
$$

Mais il reste à demander à l'observation, pour chaque inclinaison sur la verticale de la face d'amont du déversoir, le rapport à la hauteur de charge $h$ du relèvement total $\varepsilon$ que la surface inférieure de la nappe liquide éprouve en quittant le seuil du déversoir. Pour l'obtenir, j'ai communiqué les résultats théoriques précédents, en juin : 1886 , à $M$. Bazin qui commençait alors, sur le canal de Bourgogne, d'importantes 'séries d'expériences relatives aux déversoirs, et le savant hydraulicien de Dijon, ocçupé justement à une étude à ta fois propice et très en grand, de déversoirs verticaux en mince paroi sans contration Jatérale, qui doivent lui servir d'étalon pour jauger les autres genres de déversoirs, a bien voulu pratiquer, à côté de son barrage, une chambre avec paroi en verre, permettant de voir le dessous de la nappe. Or, en traçant sur cette paroi latérale transparentc 
des traits, les uns horizontaux, les autres verticaux, il a pu, non seulement constater le fait du relèvement de la face inférieure de la nappe, qui avait, paraît-il, échappé jusqu'ici aux hydrauliciens; mais encore en mesurer très approximativement la valeur $\varepsilon$. Il a ainsi trouvé que le rapport $\frac{\varepsilon}{h}$ était à fort peu près de 0,13 dans le cas d'un déversoir ayant sa face d'amont verticale. La valeur théorique (r3) de $m$ sera donc 0,5216 . $(0,87)^{\frac{3}{2}}$, ou 0,423 , et c'est bien, en effet, à quelques millièmes près, le coefficient de débit que de nombreuses observations, dans les conditions les plus diverses, ont donné pour ces déversoirs.

\section{Sur la théorie des déversoirs en mince paroi et à nappe, soit déprimée, soit soulevée, c'est-à-dire soumise inférieurement à une pression constante, plus petite ou plus grande que celle de l'atmosphère exercée au-dessus $\left({ }^{\star}\right)$.}

I. - J'ai montré, dans une note du 4 juillet, comment la théorie des déversoirs en mince paroi, sans contraction latérale et à nappe libre, peut être édifiée sur la supposition naturelle de filets parallèles à l'endroit où ils traversent la section contractée, c'est-à-dire y ayant sur toute l'épaisseur de la nappe un centre commun de courbure, avec assimilation du relèvement total $\varepsilon$ qu'éprouve, près de la paroi dont elle s'est détachée, la face inférieure de la nappe, à la contraction d'une veine issue d'un orifice; en sorte que le rapport de ce relèvement $\varepsilon$ à la hauteur $h$ (au-dessus du seuil) du niveau d'amont, hauteur qui mesure en quelque sorte le diamètre correspondant de l'orifice proposé, soit, pour chaque forme du barrage, un véritable coefficient constant dẻ contraction, à déterminer par l'expérience.

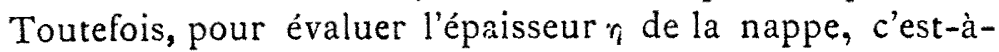
dire la hauteur de la section contractée et, par suite, le débit $q$ de l'unité de longueur du déversoir, il faut, à l'expression de $q$, de la forme $q=\mathrm{F}\left(h, r_{i}\right)$, déduite au moyen de ces hypothèses de l'équation de D. Bernoulli, appliquer un principe de débit maximum, imaginé par Bélanger, qui donne, comme relation entre $\eta$ et $h$, l'égalité à zéro de la dérivée de $q$ en $r_{i}$.

Je commencerai ici par motiver ce principe, ou plutôt ce postulatum, en imaginant que, sur le cours d'eau à débit constant dont il s'agit, le niveau d'aval ait été, d'abord, tenu assez haut pour noyer le déversoir, et puis, abaissé peu à peu, jusqu'à production, sur le barrage, du régime désormais invariable que l'on étudie, c'est-à-dire jusqu'au point à partir duquel les abaissements ultérieurs cessent de se propager sur la section contractée et, surtout, de remonter la chute précédant cette section. L'approche d'un tel état limite, où s'évanouit l'influence, sur $h$, du niveau d'aval, doit naturellement être annoncée par une atténuation indéfinie de cette influence, non seulement entre l'aval et la section contractée, mais aussi entre la section contractée et l'amont du déversoir. Donc, une dernière diminution, $-\mathrm{d}(\varepsilon+\eta)$, de la hauteur $\Sigma+\eta$ de l'eau (au-dessus du seuil) dans la section contractée n'aura provoqué qu'une dimi-

(*) Séance du ro ostobre 1887 . nution - $\mathrm{d} h$ infiniment plus faible de la hauteur $h$ d'amont: et le rapport de $\mathrm{d} h$ à $\mathrm{d} \eta+d \varepsilon$ aura été nul au moment ou s'établissait le régime dont on cherche les lois. C'est dire que, dans la relation $q=\mathrm{F}(h, \eta)$, obtenue entre $\eta$ et $h$ pour tous les états par lesquels passe l'écoulement sans que $q$ varie, il faut choisir $\eta$ de manière à annuler le rapport de $\mathrm{d} h$ à $\mathrm{d} \eta_{1}+d \bar{d}$, ou simplement de $\mathrm{d} h$ à $\mathrm{d} \eta_{i}$, vu la propor. tionnalité admise de $\varepsilon$ à $h$ et de de à d $h$.

Or, égaler ainsi à zéro la dérivée de $h$ par rapport à dans la relation $q=\mathrm{F}(h, \eta)$, où l'on suppose $q$ invariable, c'est, d'après le principe de Fermat, rendre $h$ minimum, ou choisir pour niveau d'amont le plus bas qui soit capable de fournir le débit exigé $q$ :

Et la relation :

$$
\frac{\mathrm{d} q}{\mathrm{~d} \eta}=-\frac{\mathrm{d} q}{\mathrm{~d} h} \frac{\mathrm{d} h}{\mathrm{~d} \eta}
$$

déduite de $q=$ const., revient bien alors, par la disparition de son second terme, à annuler la dérivée de $q$ en n, oúa rendre le débit $q$ maximum pour une hauteur donnée d'amont $h$, conformément à l'idée qu'à eue Bélanger.

II. - Mais, il peut arriver qu'une pression constante, - $n_{\rho} g(h-\varepsilon)$, ayant par unité d'aire un certain rapport, - $n$, au poids d'une colonne de liquide de la hauteur connue $h-\varepsilon$, s'exerce sous la nappe, et la déprime ou la soulère suivant que ce sera une non-pression ou une pression proprement dite, હ'est-à -dire un déficit ou un excédent par rapport à la pression atmosphérique dont on fait abstraction. La nappe sera donc déprimée si $n$ est positif (ce qui arrive assez fréquemment), soulevée si $n$ est, au contraire, négat if. Le rapport de $\varepsilon$ à $h$ se trouve probablement diminué dans le premier cas et accru dans le second; mais, comme sa valeur pour le cas intermédiaire $n=0$ est assez forte (o, 14 environ), il est présumable que sa partie variable: d'une forme telle que - $A n$, reste, souvent, peu sensible en comparaison. Il suffit, d'ailleurs, que rapport ce soit, pout chaque forme de déversoir, uniquement fonction du nombre donné $n$ caractérisant la non-pression exercée sous la nappe, ou même, d'une manière plus générale, que le relèvement de la face inférieure de celle-ci se trouve déterminé dès que $h$ et $n$ le sont, pour que la même méthode conduise à une expression de $q$ encore fonction des deux seules variables distinctes $h, \eta$, et comportant, par suite, en vertu du raison. nement précédent, l'application du principe de débit maximum.

La marche à suivre est identiquement celle de ma note du 4 juillet, dont j'adopterai ici les notations et les calculs. Rien n'y sera changé jusqu'à la formule (4), qui, à raison de la

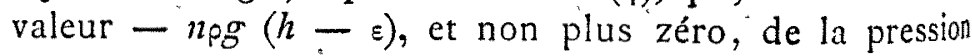
exercée sur la face inférieure de la nappe, deviendra:

$$
V=\sqrt{2 g(h-\varepsilon)(\mathrm{I}+n)} \frac{R_{0}}{R_{0}+z-\varepsilon}
$$

Et l'on sera conduit ensuite, en appelant $k$ une quantité comprise entre zéro et l'inverse de $\sqrt{\mathrm{I}+n}$ quand $\eta$ l'est entre $h-\varepsilon$ et zéro, à poser :

$k=\sqrt{\frac{h-\varepsilon-\eta}{(h-\varepsilon)(\mathrm{I}+n)}}$ ou $\frac{\eta}{h-\varepsilon}=1-\mathrm{k}^{2}(1+n)$ 
$\frac{R_{0}}{q}=\frac{k}{\mathrm{I}-k}$; d'où il résultera, pour le débit $q$, la formule :

$$
q=\left\{\begin{array}{c}
\sqrt{2 g(h-\varepsilon)(\mathrm{I}+n)} R_{0} \log \cdot \frac{R_{0}+n}{R_{0}} \\
=\sqrt{2 g}(h-\varepsilon)^{\frac{3}{2}} \mathrm{f}(k)
\end{array}\right\}
$$

arec :

$$
f(k)=\left[(k \sqrt{\overline{\mathrm{I}+n}})-(k \sqrt{\mathrm{I}+n})^{3}\right] \frac{\log k}{k-\mathrm{I}}
$$

Le quotient, essentiellement positif, de $\log k$ par $k$ - I ne présente aucune discontinuité pour la valeur $k=\mathrm{r}$ (qui le rend égal ì I), et, s'il devient infini à la limite $k=0$, l'influence prépondérante du facteur entre crochets, dans l'expression de $\mathrm{f}(k)$, rend, en définitive, cette fonction $\mathrm{f}(k)$ nulle aux deux limites $k \sqrt{1+n}=0$ et $k \sqrt{\mathrm{I}+n}=\mathrm{r}$, entre lesquelles seules elle est positive et doit être considérée. Elle y a donc le maximum qu'on cherche et qui s'obtiendra en annulant la dérivée $\mathrm{f}^{\prime}(k)$. Or, l'équation $\mathrm{f}^{\prime}(k)=0$, résolue par rapport à $k^{2}(\mathrm{I}+n)$, donne l'équation (1 7 ) suivante:

$$
k^{2}(1+k)=\frac{1}{1+\varphi(k)}, \text { en posant } \frac{2}{\varphi(k)}=\frac{1}{\log k}+\frac{1}{1-k}
$$

Une valeur positive quelconque, attribuée à $k$, rendra $\mathrm{f}(k)$ maximum pour la valeur de $n$ résultant immédiatement de cette relation (17); et il suffira de faire, dans celle-ci, varier $k$, pour former le tableau des valeurs correspondantes de $n$, puis, grâce à ( 16 ) et (I 5$)$, celui des maxima $M=\mathrm{f}(k)$, ainsi que des rapports de $\eta$ et de $R_{0}$ à $h-\varepsilon$.

$\begin{array}{llllll}k=\ldots \ldots \ldots \ldots \ldots \ldots & 0 & 0, \mathrm{I} & 0,2 & 0,3 & 0,4 \\ n=\ldots \ldots \ldots \ldots \ldots & \infty & 24,28 & 4,979 & \mathrm{1}, 5575 & 0,396 \mathrm{r} \\ \frac{\eta}{h-\varepsilon}=\ldots \ldots \ldots \circ, 6667 & 0,747^{2} & 0,7608 & 0,7698 & 0,7766 \\ \frac{R_{0}}{h-\varepsilon}=\ldots \ldots \ldots \ldots \ldots & 0,0830 & 0,1902 & 0,3299 & 0,5177 \\ M \text { ou } \frac{q}{\sqrt{2 g}(h-\varepsilon)^{\frac{3}{2}}}=\infty & 0,961 \mathrm{I} 2 & 0,7486 & 0,6352 & 0,5605\end{array}$

III. - Pour voir comment se succéderont les valeurs de $n$, étudions, dans ( 17 ), la fonction $\varphi(k)$, qui, égale à 2 pour $k$ nul et à $2 \log k$ (sensiblement) pour $k$ très grand, ne cesse pas d'être continue pour la valeur $k=\mathrm{I}$, près de laquelle $\log k$, développé en série suivant les puissances de $k-1$, a comme inverse $\frac{1}{k-1}\left(1+\frac{k-1}{2}-\ldots\right)=$ $\frac{1}{1-k}+\frac{1}{2}-\ldots$, donnant ainsi $\dot{\phi}(k)=4$ à la limite $k-1$. Céte fonction $\varphi(k)$ croît sans cesse avec $k$. En effet, la deuxième relation (17), différenciée, montre que que $\varphi^{\prime}(k)$ a le signe de $(\mathrm{r}-k)^{2}-k(\log k)^{2}$, fonction essentiellement positive; car, continue avec ses dérivées, pour $k>0$, et nulle, avec șa dérivée première, pour $k=1$, elle a sa dérivée seconde de même signe que l'expression $k-\mathrm{I}-\log k$, dont le minimum, atteint pour $k=\mathrm{r}$, est zéro. Ainsi, lorsque $k$ grandit de zéro à l'infini, $\varphi(k)$ croît de 2 à l'infini; et, d'après ( 17$)$, le produit $k^{2}(\mathrm{r}+n)$ diminue de $\frac{1}{3}$ à zéro, ou $n$ de l'infini à - r. Mạis, la nappe déversante étant toujours concave en dessous, le rayon $R_{0}$ de courbure de sa face inférieure, sur la section contractée, ne devient jamais négatif. Donc, d'après la dernière ( 15$), k$ n'a pas à dépasser l'unité; ce qui ne laisse $\varphi_{(}(k)$ varier que de 2 à $4, k^{2}(\mathrm{I}+n)$ de $\frac{\mathrm{I}}{3} \grave{a} \frac{\mathrm{I}}{5}, n$ de $\infty \grave{\mathrm{a}}-\frac{4}{5}$, et, l'épaisseur $r_{\text {de la lame, de }}$ $\frac{2}{3}(h-\varepsilon)$ à $\frac{4}{5}(h-\varepsilon)$, c'est-à-dire du onzième seulement de sa valeur moyenne $\frac{\mathrm{II}}{15}(h-\xi)$, de part et d'autre de celle-ci, tandis que le rayon de courbure $R_{0}$ croît de zéro à l'infini.

Par suite, d'après ( $\mathrm{t} 6$ ), le rapport $\mathrm{f}(k)=M$ du débit $q$, à $\sqrt{2 g}(h-\varepsilon)^{\frac{3}{2}}$ variera surtout à raison du facteur $\frac{\log k}{k-1}$ Or, celui-ci est décroissant; car sa dérivée a le signe de l'expression $\left(\mathrm{I}-\frac{1}{k}\right)+\log \frac{1}{k}$, ou $(\mathrm{I}-x)+\log x$, qui atteint son maximuñ zéro pour $x=\mathrm{I}$. C'est à dire que le coefficient $M$ de la formule $M V \sqrt{2 g}(h-\varepsilon)^{\frac{1}{2}}$ du débit varie, comme l'on pouvalt s'y attendre, en sens inverse de $k$, et dans,le même sens que la non-pression mesurée proportionnellement par $n$. Sa valeur la plus faible correspond donc à $k=\mathrm{I}$ et à $R_{0}=\infty$, ou à une forme rectiligne des filets dans la section contractée (ce qui entraîne une distribution hydrostatique des pressioris sur cette section); elle est: $\frac{4}{5 \sqrt{\overline{5}}}=0,3578$

Voici, d'ailleurs, les résultats du calcul pour les valeurs de $k$ multiples de $\mathrm{o}, \mathrm{I}$, avec intercalation de ceux qui correspondent au cas $n=0$ de la nappe libre, et que j'avais déduits, à ma note du 4 juillet, de formules comprises dans les précédentes:

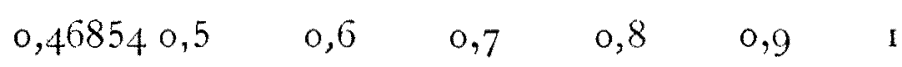

$$
\text { o } \quad-0,[283-0,4074-0,5726-0,6783-0,7494-0,8
$$
0,7805
$0,782 \mathrm{I}$
$0,7867 \quad 0,7906$
$0,794 \mathrm{I}$
$0,7971 \quad 0,8$

$\begin{array}{llllllll}0,6881 & 0,7821 & 1,1800 & 1,845 & 3,176 & 7,174 & \infty\end{array}$

$0,5216 \quad 0,5061 \quad 0,4640 \quad 0,4301 \quad 0,4020 \quad 0,3783 \quad 0,3578$

IV. - Des déterminations précises du relèvement $\varepsilon$, pour les nappes libres se détachant de déversoirs en mince paroi et à face d'amont verticale, ont été faites récemment par M. Bazin (*); elles ont donné, pour le rapport de $\varepsilon$ à $h$, comme résultats moyens, respectivement $0,142,0,136$, $\mathrm{o}, 137, \mathrm{o}, \mathrm{r} 33$, entre $h=\mathrm{o}_{2} \mathrm{~m}_{2}$ et o $\mathrm{m}_{2} 5, \mathrm{om}_{2} 5$ et om 30 , om $3 \mathrm{o}$ et $\mathrm{o}^{\mathrm{m}} 35, \mathrm{o}^{\mathrm{m}} 35$ et $^{\mathrm{m}} 42$. Pour de moindres valeurs de $h$, qui ont varié de $\mathrm{o}_{\mathrm{I}} 5$ à $\mathrm{om}_{2 \mathrm{O}}$,'ce rapport a eu la valeur moyenne, plus élevée, 0,157 ; mais on peut craindre que l'approximation n'y ait pas été suffisante sur les très petits relèvements alors observés $\varepsilon$, de $o_{0} m_{0} 3$ au plus. C'est pourquoi j'adopterai comme valeur normale du rapport de $\varepsilon$ à $h$, pour le genre de déversoirs dont il s'agit, le nombre exact de centième 0,14 , c'est-d̀-dire la moyenne $(0,137)$ des quatre premiers résultats ci-dessus, un peu forcée pour tenir compte de ce que la hauteur du déversoir $\left(\mathrm{I}_{1} 3\right)$ n'était pas indéfinie, comme on le supose, ni, par suite, la contraction

(*) Sans l'interposition de la paroi latérale en verre, dont il est parlé à la fin de ma note du 4 juillet, mais dơnt l'expérience a montré l'inutilité et les inconvénients. 
còmplete. Alors, le tableau ci-dessus donnera, dans le cas d'une nappe libre:

$$
\begin{gathered}
q=0,4160 h \sqrt{2 g h} \quad \eta=0,6712 h \\
R_{0}=0,5918 h=4,227 \approx
\end{gathered}
$$

Les expériences de M. Bazin confirment, on ne peut mieux, cette expression de $g$. Elles n'ont pas encore porté sur l'épaisseur $r_{1}$, dont le mèsurage sera d'ailleurs, à ce qu'il semble, assez aisé. Mais la confrontation serait plus difficile en ce qui concerne le rayon de courbure $R_{0}$ du dessous de la nappe dans sa partie la plus haute. On peut, toutefois, contrôler jusqu'à un certain point sa valeur théorique $4,227 \varepsilon$ grâce au tracé qu'a eu soin de fäire M. Bazin sur plusieurs profils en long de cette surface observés par lui, de la corde horizontale émanée du seuil. Sa longueur a été trouvée cónstamment égale à $5 \varepsilon$, dont un tiers, soit environ $1,7 \varepsilon$, en deça de la flèche $\varepsilon$, et les deux autres tiers, ou $3,3 \varepsilon$, au delà. Or, l'arc de cette corde, évidemment vertical au départ comme la vitesse ascendante des filets fluides qui le décrivent et qui ont glissé sur la face amont $\mathrm{d}$ a déversoir, tourne d'abord très vite, pour devenir horizontal au sommet de la flèche $\varepsilon$, et présente ainsi des courbures de plus en plus lentement décroissantes, jusqu'à la seconde extrémité de la corde considérée $5 \varepsilon$. Donc, cette même corde, mais prolongée en deça du seuil, et supposée terminée de part et d'autre à la circonférence $2 \pi R_{0}$ osculatrice au sommet, aura, de chaque côté de la flèche $\varepsilon$, une longueur, $V \overline{\left(2 R_{0}-\varepsilon\right) \varepsilon,}$ excédant notablement plus $\mathrm{I}, 7 \varepsilon$, vers le seuil, qu'elle ne sera inférieure à $3,3 \varepsilon$, au de la flèche. Or, c'est précisément ce qui résulte de la valeur théorique $R_{0}=4,227 \varepsilon$; car omen déduit :

$$
V \overline{\left(2 R_{0}-\varepsilon\right) \varepsilon}=2,730 \equiv .
$$

V. - Il est clair, par les mêmes considérations, que, dans un déversoir à nappe déprimée, le rapport de $R_{0}$ à $\varepsilon$ ne pourrait décroître jusqu'à une limite telle que l'unité, ou le centre du cercle $2 \pi R_{0}$ osculateur au point le plus haut de la face inférieure de la nappe s'élever environ jusqu'au niveau du seuil et même seulement en approcher, sans que, par le fait même, cette face redevînt, en descendant, verticale dès ce niveau ou un peu au-dessous, ne laissant ainsi, entre elle et lé déversoir, qu'une mìrce couche d'air, d'une épaisseur à peine supérieure à $2 \varepsilon$, et dont l'entraînement, dès lors inévitable, amènerait soit l'adhérence de la nappe à la face aval du barrage, soit du moins lintroduction, contre cette face, d'une couche d'eau tourbillonnante. et d'étendue notable noyant an dessaus ta nappe de déversement. Si l'on admet provisuirement que, meême pour d'assez grándes vafeurs de $n$, le quotient de $\varepsilon$ par $h$ reste à peu près $0, \mathrm{~F} 4$, ou, celui de $\varepsilon$ par $h-\varepsilon, \sigma, \mathrm{I} 63$, la mappe devra donc devenir ou adhérênte, au noyée en dessous, quand le rapport de $R_{0}$ à $h-r e$ diminuera jusqu'à ne plus dépasser beaucoup 0,163 . D'aprés la surite des valeurs de ce rapport contenures dans le tableau précédent, aư 'l'on' voit qu'il se trauve déjà réduit à o, 190 lorsque $k$ descend jusqu'à o,2, ou lorsque $n$ atteint 5 environ, on peut conjecturer que l'expulsion de l'air et même l'adhénence de la nappe sont dès lọ̣s effectuées، Ces phénomènes remarquables, signalés par $M$. Bazin comme correspondant à des changements profonds du mode de l'écoulement et dit bruit qui l'accompagne, surviendraient donc quand la non. pression sous-la-nappe (évaluée en hauteur d'eau), appro. cherait de $5(h-\varepsilon)$ ou $4,3 h$, c'est-à-dire deviendrait voisine de 4 fois entriton la hauteur de charge $h$. Alors, le coefficient de débit $m=M(1-0,14)^{\frac{3}{2}}$, rapport de $q$ à $h \sqrt{2 g h}$, approcherait lui-même, d'après le tableau précédent $_{,}$de la valeúr considérable $0 ; 7486(o, 86)^{\frac{3}{2}}=0,6$ environ.

Si l'on continue à admettre provisoirement que $\varepsilon=0,14 \mathrm{~h}$ pour ce cas, et pour ceux de non-pressions moindres, ou encore de pressions positives modérées exercées sous la nappe, le même tableau des valeurs de $n$ et de $M$ donnera:

$\begin{array}{llllll}\text { Pour } k=0,2 \quad 0,3 & 0,4 & 0,5 & 0,6 & 0,7\end{array}$ ou pour les non pressions :

$$
m\left(^{*}\right)=\begin{array}{llllll}
4,282 h & 1,33 y h & 0,341 h & -0,110 h & -0,350 h & -0,492 h \\
0,597 & 0,507 & 0,447 & 0,404 & 0,370 & 0,343
\end{array}
$$

les nombres de la deuxième colonne étant les valeurs de :

$$
n(h-\varepsilon)=n(1-0,14) h
$$

et, ceux de la troisième, les valeurs de $M(1-0,14)^{\frac{3}{2}}$.

(A sulvre.)

\section{LE MOIS HYDRO-ÉLECTRIQUE}

\section{ACADÉMIE DES SCIENCES}

\section{MÉGANIQUE ET ÉLEGTRIGITÉ}

Emploi des flammes comme soupape des courants alternatifs à haute tension. - Notes de M. André CATHIARD, séances dill 6 janvier et du 2 mars 1908.

Si l'on présente à une flamme, ne contenant aucun corps conducs teur solide en suspension, deux électrodes quelconques, l'unè di surface très petite par rapport à l'autre (section d'un fil ou d'une tıge), et toutes deux reliees à une source alternative à haute tension, on constate le passage d'un courant de même sens, allant à travers la flamme de la grande électrode vers la petite.

Le phénomène est très accentue si l'on prend comme petite élettrode un conducteur de forme effilée.

Dans ces conditions, il se produit dans la flamme une sorte d'ar très peu lumineux, et dont le point de contact sur la grande électrode, qui est pasitive, est en mouvement.

Les expériences ont porté sur plúsieurs sortes d'électrodes et de flammes, hydrogène, alcool, gaz d'éclairage et, en particulier, sut cette derntëre, obtenue par ün bec Mecker de 300 litres à l'heure, avec électrodes en charbon graphitique. La petite surface était constituée par la section cylindrique d'une baguette c ce charbon de $5 \mathrm{~mm}$. de diamètre. Tensions de 2000 à ro ooo volts obtenues par: un transformateur, fréquence de 40 périodes par seconde, distánce entre électrodes variant de $5 \mathrm{~mm}$ à $10 \mathrm{~cm}$., dans le thême plan, ol dans'des plans différents perpend culaires à la flamme.

Si l'on retirait la petite électrode de la tlamme, le phênomène restait le même jusqư'à devenir nul lorsque cette électrode était trop éloignée.

L'intensité moyenne du courant, mesurèe par un ampèrémétre: càdre mobile, n'a jamais dépassé 0,03 ampère. Au dessus, il avaît formation d'un arc brillant, avec transport de carbone solide et le phénomène 'cessàit.

bes relevés oscillographiques n'ont pas encore été effeçtués, misis disons de suite, à titre de renseignement, que le courant' était suffsamment de même sens pour permettre fobtention des dépôts galvă niques.

$\stackrel{4}{*}$ La valeur de mest $m=\frac{q}{h \sqrt{2 g h}} m$. 